\title{
Could Telehealth Improve Equity During the COVID-19 Pandemic?
}

Cynthia J. Sieck, PhD, MPH, Mark Rastetter, MD, and Ann Scheck McAlearney, ScD, MS

In response to the COVID-19 pandemic, many physicians and health care systems have shifted to providing care via telehealth as much as possible. Although necessary to control spread of the virus and preserve personal protective equipment, this shift highlights existing disparities in access and care. Patients without the skills and tools to access telehealth services may increase their risk of exposure by seeking care in person or may delay care entirely. We know that patients need internet access, devices capable of visual communication, and the skills to use these devices to experience the full benefits of telehealth, yet we also know that disparities are present in each of these areas. Currently, federal programs have given physicians greater flexibility in providing care remotely and have expanded internet access for vulnerable patients to promote telehealth services. However, these changes are temporary and it is uncertain which will remain when the pandemic is over. Family medicine physicians have an important role to play in identifying and addressing these disparities and facilitating more equitable care moving forward. (J Am Board Fam Med 2021;34:S225-S228.)

Keywords: COVID-19, Family Medicine, Health Equity, Internet Access, Pandemics, Internet Telemedicine

The COVID-19 pandemic necessitated a rapid shift from in-person visits to the use of telehealth to limit the spread of the virus. Yet we know that the impact of a switch to telehealth is not the same for all populations. This pandemic has both highlighted and exacerbated existing disparities in access and care. ${ }^{1-4}$ We must therefore make efforts to understand the needs and readiness of our patient population to use telehealth modalities as well as ensure proper mechanisms are in place to support their use. What we learn now about responding to disparities in care and access can shape a new, more equitable normal in health care.

This article was externally peer reviewed.

Submitted 14 May 2020; revised 9 July 2020; accepted 15 July 2020.

From the Department of Family and Community Medicine, College of Medicine, Ohio State University; and CATALYST, Center for the Advancement of Team Science, Analytics, and Systems Thinking, College of Medicine, Ohio State University.

Conflict of interest: The authors have no funding or conflicts of interest to report.

Corresponding author: Cynthia J. Sieck, PhD, MPH, Associate Professor of Family Medicine, CATALYST, Center for the Advancement of Team Science, Analytics, and Systems Thinking in Health Services and Implementation Science Research, College of Medicine, Ohio State University, 460 Medical Center Drive, Suite 524, Columbus, OH 43210,
Patients Have Different Experiences With Stay-atHome Orders and Other Recommendations Designed to Reduce Risk of Exposure

We know that some groups face greater challenges in complying with stay-at-home orders. People living in population-dense areas are less able to practice physical distancing. Those who cannot afford 2 weeks of groceries must shop more frequently, increasing their risk of exposure. Essential workers reliant on public transportation face greater risks than those who can drive their own cars. Patients without the technology and/or skills to access telehealth services may risk in-person visits when not necessary for their medical condition, rely on more limited communication methods to interact with their providers, or simply delay care. Even when inperson care is possible, telehealth options will remain, but some populations will still be at higher risk of infection or have concerns about seeking inperson care. ${ }^{5}$ Understanding equitable access to telehealth options is critical to the ability of family medicine physicians to plan an appropriate course of care for these patients.

United States. Phone: (614) 366-0218; Fax: (614) 293-2715, (E-mail: Cynthia.sieck@osumc.edu). 


\section{The Right Tools, Access, and Skills Are Necessary for Full Use of Telehealth}

Patients need a device that supports audio and visual communication and access to home internet to communicate using that device. Telephone consultations are useful; however, visual communication expands assessment opportunities. ${ }^{6}$ There are also a number of tools that support remote health care delivery that rely on access to appropriate medical devices and technologies. For example, remote symptom-monitoring tools such as blood pressure cuffs and pulse oximeters may reduce the need for in-person visits. However, access to such tools and individuals' abilities to remotely transmit the data to a primary care office are not the same across the United States. Whereas $81 \%$ of the US population overall owns a smartphone, that percentage drops to $53 \%$ for older adults. ${ }^{7}$ Furthermore, those with smartphones may have limited data plans or may share a device across family members, making reliance on this device for telehealth a burden. In addition, nearly $40 \%$ of low-income households and $37 \%$ of rural households lack a broadband internet subscription. $^{8,9}$ Whereas programs are available to connect patients to needed devices and the internet, ${ }^{10}$ screening patients to ensure they have both internet and device access will remain critical.

\section{Digital Skills and Literacy Are in Addition Necessary}

Literacy, the ability to read and write, and health literacy, the ability to obtain, process, understand, and communicate health-related information, are critical skills to engaging in one's health care. ${ }^{11}$ Digital literacy in the context of health care extends these concepts and refers to the ability to access and understand health information online and use health information technology tools to participate in one's health care. Tools to screen for digital literacy can identify who needs help and what type of help. Some patients may need help learning to use any technology, whereas others may be able to use a computer or smartphone but need training to use specific tools. Currently, digital literacy training is available from a variety of sources including libraries, community centers, and others-and many programs have quickly adapted in this pandemic to provide remote access to their services. ${ }^{10}$ Partnering with these organizations now and in the future may preserve the ability to screen patients for digital literacy as well as target needed training focused on the use of telehealth tools-important steps toward reducing disparities in access to and use of telehealth. In the current pandemic situation, providers are using multiple platforms out of necessity, but these platforms vary for both patients and providers in terms of usability as well as integration with electronic health records. Moving forward, examining usability and ensuring telehealth platforms work for both these groups is key to ensuring a sustainable, equitable system.

\section{Partnerships in Vulnerable Communities Can Help Address Needs}

Recent policy articles highlight how multistakeholder partnerships can address a patient's broader needs to maintain their health. For example, health care systems and social services are becoming increasingly integrated through the use of social workers and community health workers, the incorporation of social risk screening into medical care, and the introduction of polices that expand coverage for social services. ${ }^{12}$ Specific to older adults, partnerships between area agencies on aging and health care organizations can support transitions from hospital to home. ${ }^{13}$ Partnerships such as these can also be leveraged to increase digital literacy and access by linking patients in need of training, access, and devices with appropriate organizations. Notably, these partnerships can help to meet immediate needs in this critical time as well as serve patients made vulnerable well into the future.

\section{Policy Changes Facilitate Access Now, but They Are Temporary}

Currently health care providers have significant flexibility in how they are able to remotely interact with patients. Restrictions on software used for telehealth visits as well as policies related to documentation have been relaxed in the face of the need to get patients and providers online quickly. ${ }^{14}$ Whereas few would advocate for all relaxed policies to remain, we must consider how such policies may benefit at-risk populations. The Federal Communications Commission is offering grants to health care systems allowing them to pay for internet services and devices for patients and has temporarily allowed use of additional space in the broadband spectrum to support the increase in internet use. ${ }^{15}$ Many internet service providers agreed to temporarily suspend service terminations, waive late fees, and open local WiFi hotspots as well as having expanded their offerings to 
lower-income households and adjusted eligibility requirements during the pandemic. ${ }^{10}$ These changes are helping children stay connected to their school work, helping employees continue to work remotely, and helping patients use telehealth services. However, these options are in place only until September 30, 2020, begging the question of what happens next?

\section{Family Medicine Physicians Play a Vital Role in Promoting Digital Equity to Improve Their Patients' Health and Health Care Experience}

Telehealth care has quickly gained ground in this pandemic, and it calls on the ingenuity, flexibility, and patient-centered mentality of the family medicine physician to assure our patients are receiving equitable and high-quality care both now and moving forward. Advocating for office practices that allow health literacy screening, as well as digital literacy screening, can help reduce disparities in health care as well as in education and employment opportunities. ${ }^{16,17}$ Whereas screening alone is not sufficient to solve issues of equity, it is a critical starting point that allows the identification and documentation of the extent of challenges and can help practices better target their limited resources. Workflows that structure these screenings outside the office visit are key to ensuring our patients, and providers are best equipped during subsequent clinical consultations. Notably, the recent surge in telehealth use has significantly increased the number of patients and physicians who now have experience using this modality of care. This experience allows family medicine physicians to view telehealth as an important location of care and understand its usefulness in addressing a variety of medical conditions. Assuring our practices are partnering with community agencies that can provide digital literacy services will allow us to both leverage our time and expertise for efficient and effective care.

Offering telehealth services comes at a cost both for patients and physician practices and particularly for outpatient care delivery. ${ }^{18}$ Calling on our national organizations to push for private, state, and federal reimbursement strategies both for the provision of telehealth services and the coverage of tools necessary for our patients and physicians, and especially for those living and practicing in rural and underserved areas, is vital for long-term viability. In this pandemic, we are learning a great deal about how to interact with each other remotely.
We have the opportunity to apply a health equity lens to this learning to ensure that the technologies, tools, and policies that remain in place moving forward are accessible to everyone.

To see this article online, please go to: http://jabfm.org/content/ 34/Supplement/S225.full.

\section{References}

1. Brooks O. COVID-19 underscores wealth and health disparities in the African American community. National Medical Association, Editor. Silver Spring (MD): National Medical Association; 2020.

2. Alessa T, Abdi S, Hawley MS, de Witte L. Mobile apps to support the self-management of hypertension: Systematic review of effectiveness, usability, and user satisfaction. JMIR mHealth Uhealth 2018;6:e10723.

3. Lau J, Knudsen J, Jackson H, Wallach AB, Bouton M, Natsui S, et al. Staying connected in the COVID-19 pandemic: Telehealth at the largest safety-net system in the United States. A description of NYC Health + Hospitals telehealth response to the COVID-19 pandemic. Health Affairs 2020; 39:1437-42. 10.1377/hlthaff. 2020.00903.

4. Totten AM, McDonagh MS, Wagner JH. The evidence base for telehealth: Reassurance in the face of rapid expansion during the COVID-19 pandemic. 2020.

5. Ross C. 'I can't imagine going back': Medicare leader calls for expanded telehealth access after Covid-19. 2020; STAT. Available from: https://www.statnews. com/2020/06/09/seema-verma-telehealth-accesscovid19/.

6. Nouri S, Khoong EC, Lyles CR, Karliner L. Addressing equity in telemedicine for chronic disease management during the Covid-19 pandemic. N Engl J Med Catal Innov Care Deliv 2020;1:3.

7. Pew Research Center. Mobile Fact Sheet. 2020. https://www.pewresearch.org/internet/fact-sheet/ mobile/.

8. Tomer A, Fishbane L, Siefer A, Callahan B. Digital prosperity: how broadband can deliver health and equity to all communities in Metropolitan Infrastructure Initiative. Brookings Institution; 2020. Available from: https://www.brookings.edu/research/digitalprosperity-how-broadband-can-deliver-healthand-equity-to-all-communities/.

9. Perin A. Digital gap between rural and nonrural America persists. 2019. Available from: https:// www.pewresearch.org/fact-tank/2019/05/31/digitalgap-between-rural-and-nonrural-america-persists/.

10. National Digital Inclusion Alliance. 2019. https:// www.digitalinclusion.org/definitions/.

11. Kindig, David A, Allison M. Panzer, Lynn NielsenBohlman, eds. Health literacy: a prescription to end confusion. National Academies Press; 2004. 
12. Fichtenberg C, Delva J, Minyard K, Gottlieb LM. Health And Human Services integration: generating sustained health and equity improvements: an overview of collaborations, partnerships, and other integration efforts between health care and social services organizations. Health Aff (Millwood) 2020;39:56773.

13. Brewster AL, Kunkel S, Straker J, Curry LA. Cross-sectoral partnerships by area agencies on aging: associations with health care use and spending. Health. Health Aff (Millwood) 2018;37:15-21.

14. Magoon V. Providing options for remote access to care can keep your practice running and your patients healthy when an infectious disease outbreak prevents you from seeing them in person. 2020. Available from: https://www.aafp.org/journals/fpm/ explore/online/virtual_visits.html.
15. Commission FC. Lifeline support for affordable communications. 2020. https://www.fcc.gov/consumers/ guides/lifeline-support-affordable-communications.

16. Reisdorf BC, Yankelevich A, Shapiro M, Dutton WH. Wirelessly bridging the homework gap: technical options and social challenges in getting broadband to disconnected students. Educ Inf Technol 2019;24:3803-21.

17. Bai Y. The faster, the better? The impact of internet speed on employment. Inform Econ Policy 2017;40: 21-5.

18. Basu S, Phillips RS, Phillips R, Peterson LE, Landon BE. Primary care practice finances in the United States amid the COVID-19 pandemic: study estimates the potential impact of COVID-19 on operating expenses and revenues of primary care practices. Health Aff 2020;39:1605-14. 